\title{
Beam transfer functions for relativistic proton bunches with beam-beam interaction
}

\author{
P. Görgen ${ }^{\mathrm{a}, *}$, O. Boine-Frankenheim ${ }^{\mathrm{a}}$, W. Fischer ${ }^{\mathrm{b}}$ \\ ${ }^{a}$ Institut für Theorie Elektromagnetischer Felder (TEMF), Technische Universität \\ Darmstadt, Schloßgartenstr. 864289 Darmstadt, Germany \\ ${ }^{b}$ Brookhaven National Laboratory, Upton, NY 11973, United States
}

\begin{abstract}
We present a method for the recovery of the transverse tune spread directly from the beam transfer function (BTF). The model is applicable for coasting beams and bunched beams at high energy with a tune spread from transverse nonlinearities induced by the beam-beam effect or by an electron lens. Other sources of tune spread can be added. A method for the recovery of the incoherent tune spread without prior knowledge of the nonlinearity is presented. The approach is based on the analytic model for BTFs of coasting beams, which agrees very well with simulations results for bunched beams at relativistic energies with typically low synchrotron tune. A priori the presented tune spread recovery method is usable only in absence of coherent modes, but additional simulation data shows its applicability even in presence of coherent beam-beam modes. Finally agreement of both the analytic and simulation models with measurement data obtained at RHIC are presented. The proposed method successfully recovers the tune spread from analytic, simulated and measured BTF.

Keywords: beam transfer function, tune distribution, electron lens, incoherent effects, hadron colliders, beam-beam
\end{abstract}

Transverse Beam Transfer Functions (BTF) are a powerful diagnostic tool. They are used for a wide range of applications, the most prominent of which

\footnotetext{
* Corresponding author

Email address: goergen@temf.tu-darmstadt.de (P. Görgen)
} 
is the detection of the machine tune and the measurement of the stability diagram [1]. Transverse BTFs are measured routinely using baseband Tune (BBQ) 5 systems employing CERNs direct diode detection for increased signal to noise ratio [2]. In coasting beams the BTF can also be used to detect the transverse tune spread from space charge [3] and thereby directly measure the magnitude of the transverse space charge. For bunched beams with high synchrotron frequency, head-tail modes have been observed in BTFs [4].

This paper focuses on the BTF of bunched beams with synchrotron periods of the same order of magnitude as the data acquisition time per BTF sample. We assume the synchrotron frequency is orders of magnitude below the betatron frequency, conditions commonly found in high energy machines. So far the usual practice is to look at the width of the signal amplitude in either Schottky 15 spectra or BTFs and use it as an estimate for the tune spread. Where the BTF is measured, one also uses the imaginary part of the BTF as an estimate for tune distribution. We present this method and show that it can not in general be applied to beams with a transverse nonlinearity as a source of the tune spread.

One source of tune spread and the motivation for our study is the electron lens recently installed at the Relativistic Heavy Ion Collider (RHIC) [5]. We want to be able to measure the tune spread it introduces. Other, more widespread sources of tune spread one might want to quantify using BTFs are space charge or higher order multipoles. An electron lens is a device in which a magnetically confined electron beam of defined shape is guided in parallel to 25 the ion beam in a synchrotron in order to introduce amplitude dependent focusing [6]. Comparable compensation schemes are studied for compensation of space charge [7] at RHIC and another electron lens is discussed for head-on and long-range beam-beam compensation in LHC [8]. At RHIC the electron lens will be used to partly compensate the incoherent tune spread from the beambeam interaction in proton operation [5]. We want to diagnose its effect on the tune spread using the BTF. To achieve this goal, this paper makes use of an analytic model for the BTF of a local nonlinear lens building on the existing theory for coasting beams by Berg and Ruggiero [9] based on earlier work by 
Hereward [10]. For the simulation model with Gaussian beams the tune spread and shape can be recovered by means of fitting the BTF against the presented analytic model if the shape of the nonlinearity is known. In order to treat the more general case in which the shape of the nonlinearity is not known, a method to measure the tune spread directly from the BTF is introduced. We refer to it as threshold method. The two methods are applied to analytic and simulated

40 BTFs. We proceed to discuss the beam-beam effect as a localized nonlinear lens and compare analytic results and simulations to measurements.

In the first section we discuss the analytic equation for the BTF of coasting beams undergoing an electron-lens or beam-beam interaction. The equations are valid in the limit of absence of coherent modes. We present a robust method to

45 determine tune spread for arbitrary beam shapes and nonlinearities via detection of Landau damping.

In the second section we introduce our simulation model for BTFs with the beam-beam effect and electron lenses. We compare its results to the analytic expectations and obtain agreement with the analytic expectation. We show that the tune spread determination method introduced in the analytic section applies also in the presence of coherent beam-beam modes in favorable conditions. We argue that the coasting beam equations stay valid when the synchrotron frequency is much lower than the betatron frequency.

In the last section we compare simulation and analytic results to measurements from a dedicated machine experiment. We find BTFs in agreement with our analytic expectations, for both the full BTF shape and the threshold method introduced in the first section.

\section{Analytic model}

In this section we introduce an analytic model based on the theory for coasting beams, which is extended to bunched beams in the following sections. We investigate methods to reconstruct the transverse tune distribution of the beam caused by a local nonlinearity in the horizontal and vertical plane. We motivate 
our approach by revisiting well-known analytic results for incoherent transverse BTFs of coasting beams with tune spread from chromaticity. We show that in this case the tune distribution can be easily recovered. With this in mind we present a model for the BTF due to a transverse nonlinearity. We show that even for a flat beam, the recovery of the tune distribution is not possible without prior knowledge of the exact shape of the nonlinearity that gives rise to the tune spread. For this reason, we then present a method for recovery of only the total tune spread, not the shape, from the BTF via detection of Landau damping. Because it detects Landau damping, the method works without prior knowledge of the source of the tune spread. Tune spread from chromaticity, octupoles, an electron lens, incoherent space charge or any other source will be detected in absence of coherent modes and external damping.

\subsection{Tune spread from chromaticity}

The transverse beam transfer function $R(\Omega)$ is defined as the fraction of the complex response amplitude $A(\Omega)$ of the beam per driving amplitude $D(\Omega)$ of a beam excited at the frequency $\Omega$. In the following we will often write $B T F$ for brevity when we in fact refer to the transverse BTF.

$$
R(\Omega)=\frac{A(\Omega)}{D(\Omega)}
$$

80 It is understood that the BTF is meaningful when taken at small amplitudes where $D$ scales linearly with $A$. Note that for our considerations we need the complex value of the BTF.

There is a good amount of research on BTF of coasting beams. One wellknown example that can be found in textbooks such as [11] is the case of beams with a tune spread originating from momentum spread and chromaticity. Assuming the density of particles in the beam $\psi$ is known as a function of the betatron frequency $\omega$, the BTF $R$ at frequency $\Omega$ can be calculated as:

$$
R(\Omega) \propto \int_{-\infty}^{\infty} \frac{1}{\omega-\Omega} \psi(\omega) \mathrm{d} \omega
$$


The pole at $\Omega=\omega$ can be accounted for by adding a small imaginary term to the denominator or by means of the residue theorem. Finally one arrives at:

$$
R(\Omega) \propto-i \pi \psi(\Omega)+\text { P.V. } \int_{-\infty}^{\infty} \frac{1}{\omega-\Omega} \psi(\omega) \mathrm{d} \omega
$$

90 Wherein P.V. denotes Cauchys principal value integral. In the case of chromaticity the BTF is described by Equation 3 and therefore the betatron frequency distribution is proportional to the imaginary part of the BTF. Measuring the imaginary part of the BTF directly gives the betatron frequency distribution in the plane of the BTF.

\subsection{Beam transfer functions due to localized transverse forces}

When the tune depends on the amplitude of the particle in the plane of the BTF excitation, the situation becomes more complicated. This is the case for nonlinear elements like octupoles or an electron lens. Here the particles' change in amplitude due to excitation leads to a consequent change in its betatron frequency which makes the treatment of the BTF less trivial. We build on work by Berg and Ruggiero for coasting beams. They derived the BTF of a beam with tune spread due to a localized octupole [9]. Their form for the BTF in the $i$ direction $R_{i}$ (with $i$ either $x$ or $y$ ) reads:

$$
R_{i}(\Omega)=c \cdot \int_{0}^{\infty} \int_{0}^{\infty} \frac{1}{\Omega-\omega_{i}\left(J_{x}, J_{y}\right)} \frac{J_{i} \mathrm{~d} \psi}{\mathrm{d} J_{i}} \mathrm{~d} J_{x} \mathrm{~d} J_{y}
$$

Wherein $c$ is a constant, $J_{x}, J_{y}$ are the transverse action angle variables, $\psi$ the distribution function in action angle variables and $\omega_{i}\left(J_{x}, J_{y}\right)$ betatron frequency as a function of these variables. $\Omega$ is the frequency at which the BTF is calculated. Note that this equation is still general and becomes octupole specific later in [9] by introduction of the $\omega_{i}\left(J_{x}, J_{y}\right)$ for an octupole. An equation for tune shift caused by a circular Gaussian charge distribution for space charge in these coordinates was given by Burov and Lebedev [12]. We use it as follows:

$$
\omega_{x}\left(J_{x}, J_{y}\right)=\omega_{0, x}+\xi_{\mathrm{bb}} \int_{0}^{1} \frac{\left(I_{0}\left(\frac{J_{x} z}{2}\right)-I_{1}\left(\frac{J_{x} z}{2}\right)\right) I_{0}\left(\frac{J_{y} z}{2}\right)}{\exp \left(z\left(J_{x}+J_{y}\right) / 2\right)} \mathrm{d} z
$$


with $\xi_{\mathrm{bb}}$ the maximum tune shift (for particles in the center of the beam), $I_{0}$ and $I_{1}$ the modified Bessel functions and $\omega_{0, x}$ the lattice tune in the $x$ direction. $\omega_{y}$ can be found by exchanging $x$ and $y$ in the righthand side of Equation 5 . The equation is equivalent to the one given in [13] for Beam-Beam evaluated for round beams. We use it as a model for a stationary electron lens which, in RHIC, is also a Gaussian charge distribution. For $\psi$ we use a Gaussian beam [9]: $\psi\left(J_{x}, J_{y}\right)=\sigma^{-4} \exp \left(-\sigma^{-2}\right) \exp \left(-J_{x}-J_{y}\right)$ unless noted otherwise.

If needed, chromaticity and other sources of tune spread can be added to Equation 4 by inserting its contribution to $\omega_{i}$ and $\psi$ and adding an integration over momentum if necessary.

\subsection{Flat beam case}

Compared to the case of tune spread due to chromaticity, the recovery of the tune spread from transverse sources is much harder, mostly due to the twodimensional nature of the problem: The particle tune is a function of both $J_{x}$ and $J_{y}$ and there is no way for the BTF to directly determine $J_{x}$. Generally the equitune lines will not be parallel to the coordinate axes in $J_{x}, J_{y}$, making it impossible to associate one $J_{x}$ to one tune shift in the full 2D case. To make the problem more tractable one may first assume a very flat distribution. This would for example be the case for a beam of very low vertical emittance. Say

$$
\psi\left(J_{x}, J_{y}\right)= \begin{cases}\varepsilon^{-1} \psi_{x}\left(J_{x}\right) & J_{y}<\varepsilon \\ 0 & J_{y} \geq \varepsilon\end{cases}
$$

with $\epsilon$ sufficiently small that all particles of an amplitude $J_{x}$ share the same eigenfrequency $\omega_{x}$ in $x$ direction. For this flat beam we can solve Equation 4 analytically in both directions.

\subsubsection{Horizontal BTF}

In this case we can do the integration in the $J_{y}$ direction directly (the integrand is independent of $J_{y}$ ). We arrive at $R_{x}(\Omega)$ given by:

$$
R_{x}(\Omega)=c \int_{0}^{\infty} \frac{1}{\Omega-\omega_{x}} J_{x} \frac{\mathrm{d} \psi}{\mathrm{d} J_{x}} \mathrm{~d} J_{x}
$$


We substitute $\omega_{x} \rightarrow u$ to solve the integral. We make the assumption that $\omega_{x}\left(J_{x}\right)$ is invertible on areas with non-zero $\frac{\mathrm{d} \psi}{\mathrm{d} J_{x}}$ and therefore we can express $J_{x}$ as a function of $\omega_{x}$ for all $J_{x}$. With $\frac{\mathrm{d} J_{x}}{\mathrm{~d} u} \mathrm{~d} u=\mathrm{d} J_{x}$ We get

$$
R_{x}(\Omega)=c \int_{\omega_{\min }}^{\omega_{\max }} \frac{1}{\Omega-u} J_{x} \frac{\mathrm{d} \psi}{\mathrm{d} J_{x}} \frac{\mathrm{d} J_{x}}{\mathrm{~d} u} \mathrm{~d} u=c \int_{\omega_{\min }}^{\omega_{\max }} \frac{1}{\Omega-u} J_{x} \frac{\mathrm{d} \psi}{\mathrm{d} u} \mathrm{~d} u .
$$

Noting the resemblance to Equation 3 we arrive at:

$$
R_{x}(\Omega)=c \int_{\omega_{\min }}^{P \cdot V \cdot} \omega_{\max } \frac{1}{\Omega-u} J_{x} \frac{\mathrm{d} \psi}{\mathrm{d} u} \mathrm{~d} u+i \pi \cdot c J_{x}(\Omega) \frac{\mathrm{d} \psi}{\mathrm{d} \omega}(\Omega)
$$
use its inverse and a measurement of $\psi\left(J_{x}\right)$ via an ionization profile monitor to calculate the tune distribution directly without need for a BTF measurement.

\subsubsection{Vertical BTF}

Now let us look at the vertical BTF

$$
R_{y}(\Omega)=c \iint_{0}^{\infty} \frac{1}{\Omega-\omega_{y}\left(J_{x}, J_{y}\right)} \frac{J_{y} \mathrm{~d} \psi}{\mathrm{d} J_{y}} \mathrm{~d} J_{x} \mathrm{~d} J_{y}
$$

distribution we get $\frac{\mathrm{d} \psi}{\mathrm{d} y}=-\psi_{x} \delta(\varepsilon-y)$ with $\delta$ the Dirac delta function. Therefore we can directly do the integration in the $J_{y}$-direction and arrive at:

$$
R_{y}(\Omega)=c \int_{0}^{\infty} \frac{1}{\Omega-\omega_{y}\left(J_{x}, \varepsilon\right)} \psi\left(J_{x}\right) \mathrm{d} J_{x}
$$

Which, using the same steps as before results in an imaginary part of the BTF of:

$$
\operatorname{Im}\left(R_{y}(\Omega)\right)=c \pi \psi(\Omega) \frac{\mathrm{d} J_{x}}{\mathrm{~d} \omega_{y}}(\Omega)
$$




$$
\frac{1}{\Omega-\omega\left(J_{x}, J_{y}\right)},
$$

will show a pole where $\omega=\Omega$. Therefore it is to be expected that these regions contribute to the imaginary part of the BTF. Secondly the derivative

$$
\frac{\mathrm{d} \psi}{\mathrm{d} J_{i}}
$$

replaces $\psi$ in the direction of the BTF. Therefore we expect the contributions of areas with high phase space density gradient $\mathrm{d} \psi / \mathrm{d} J_{i}$ to have a strong contribution. Compare this to the BTF of beams with tune spread from chromaticity alone (Equation 3) where density $\psi$ stands instead of its derivative. Lastly the whole integrand is multiplied by the $J_{i}$ coordinate in the direction of the BTF. Therefore particles with high amplitudes $J_{i}$ in the direction of the BTF $i$ contribute stronger to the BTF than those with low amplitudes.

180

We can apply these considerations to interpret the BTF shown in Figure 1. The influence of the large derivative at the edges of the hole on the imaginary 
part of the BTF is obvious: In the $x$ direction, the edges of the hole are nearly parallel to the lines of equal tune. Thus, the high derivative at the edge of the hole adds up at the corresponding tune. We see a corresponding peak in the imaginary part of $R$. The $y$-direction is a bit less intuitive: One expects signal at all tunes as all equi-tune lines pass areas with non-zero density gradient. For tune lines crossing only the right edge of the hole, the sign of the derivative at the edge of the hole is opposite to the sign outside. The two cancel one-another and lead to the near-zero-amplitude region in the imaginary part of the vertical BTF. For equitune lines that cross both edges of the hole, the derivatives at the edges cancel partly, and together with the contribution of the particle distribution give a finite imaginary part again. The grey line in the BTF corresponds to a phase space of a purely Gaussian beam without a hole to emphasize the change due to the hole. In the vertical BTF the difference indicates that the hole influences the BTF over its whole range. Note that the hole ranges from approximately $\Delta Q_{y} / Q_{\max }=-.55$ at its bottom left corner to $\Delta Q_{y} / Q_{\max }=-.02$ at its top corner.

In order to validate that our model is realistic we compare our results to particle in cell (PIC) tracking simulations of beams influenced by a Gaussian charge distribution. The simulation model will be discussed in detail in the second section. The results can be found in Figure 2. The simulated BTF agrees with the analytic BTF. The nonlinear nature of the particle motion under influence of a Gaussian lens does however quickly deteriorate the initial distribution chosen for PIC simulation, accounting for the softer edges of the hole in the simulated BTF and additional noise.

\subsection{Threshold method for determination of tune spread}

Disregarding the shape of the tune distribution we can investigate Equation 4 more closely: The result of $R_{i}(\Omega)$ will be purely real if for all $J_{x}$ and $J_{y}$ either $\frac{J_{x} \mathrm{~d} \psi_{i}}{\mathrm{~d} J_{i}}=0$ or $\omega_{i}\left(J_{x}, J_{y}\right)-\Omega \neq 0$. The reason is that in these cases there is no singularity in the integral. We write this as a logic expression:

$$
\forall J_{x}, J_{y}: \frac{\mathrm{d} \psi}{\mathrm{d} J_{i}}=0 \quad \vee \quad \Omega-\omega_{i}\left(J_{x}, J_{y}\right) \neq 0 \Rightarrow \operatorname{Im}\left(R_{i}(\Omega)\right)=0
$$




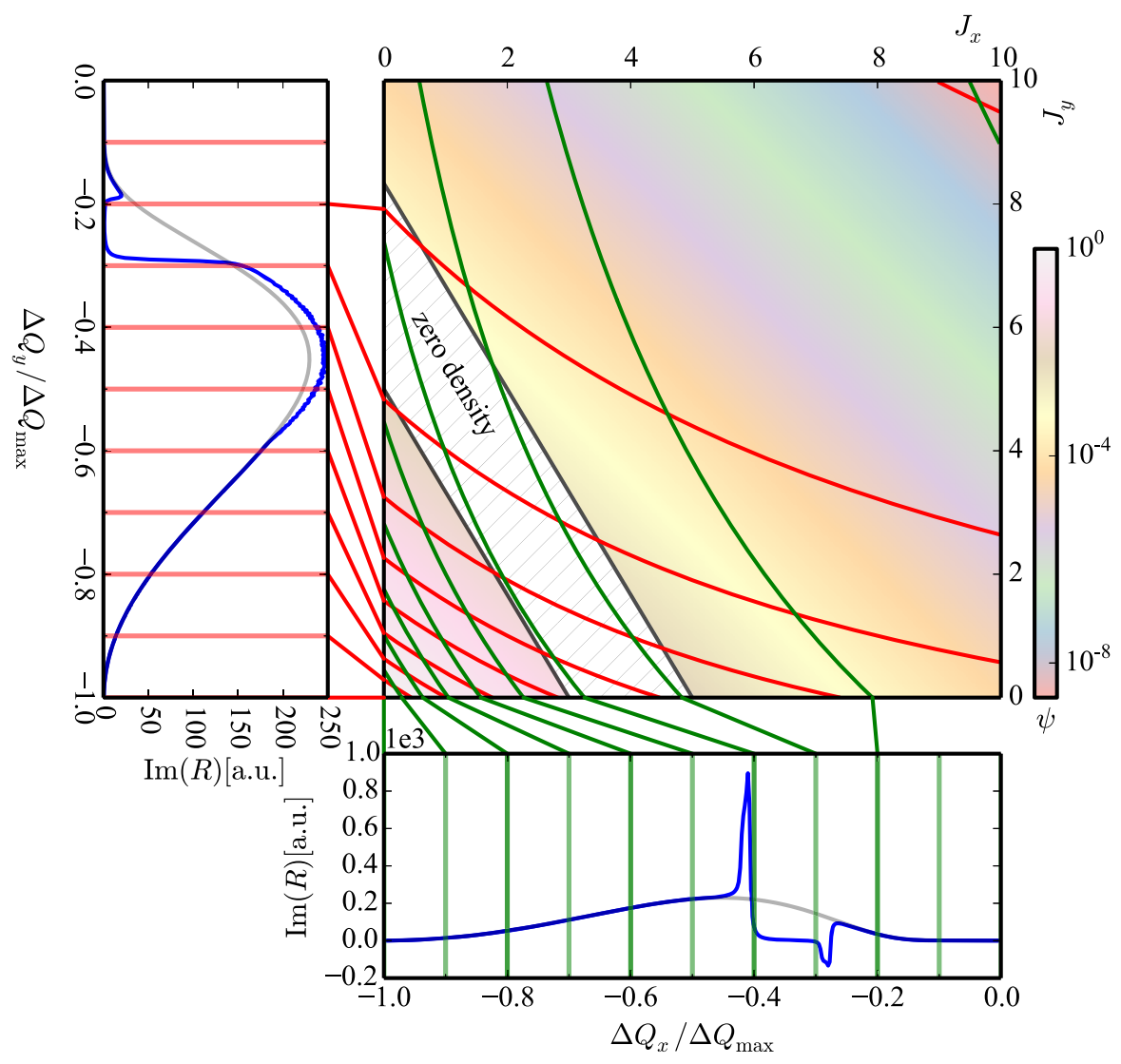

Figure 1: Example of the relations between phase space density and the resulting BTF: A hypothetical particle distribution in $J_{x}, J_{y}$ space is shown as a density plot. The density distribution $\psi$ is that of a Gaussian beam of the form $\exp \left(-J_{x}-J_{y}\right)$ in terms of $J$. It was modified by setting $\psi$ to zero for $3<J_{x}+0.6 \cdot J_{y}<5$ in order to generate a hole in phase space. Green and red lines denote contours of equal tune shift in $x$ and $y$ direction. Their numerical tune shift values can be found via the connection to the tune axes of the BTF. The BTF in $x$ and $y$ direction is shown to the bottom and to the left of the density plot. 


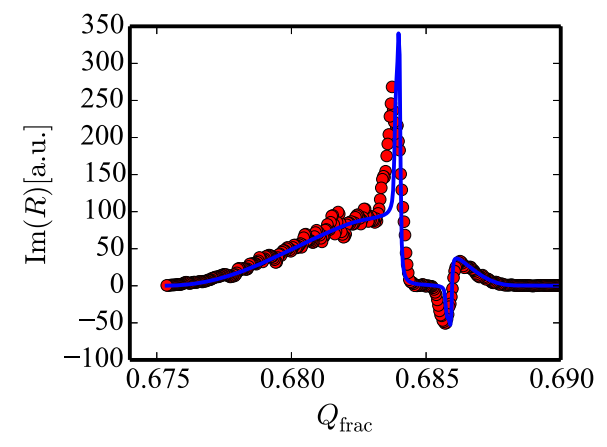

(a) BTF in $x$ direction

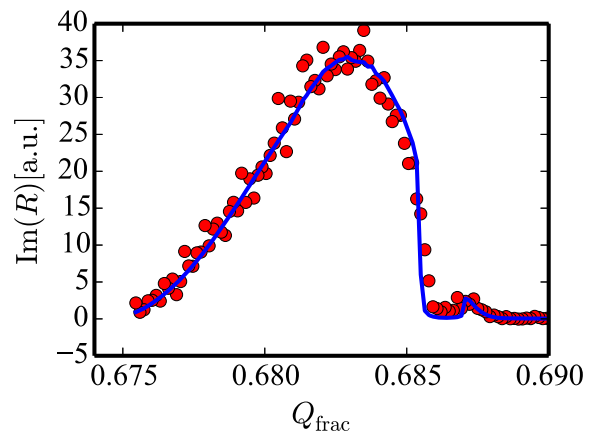

(b) BTF in $y$ direction

Figure 2: The BTF from PIC simulation (dots) agree with the analytically calculated ones (solid line) for a hole in action-angle phase space as used for Figure 1. The BTF were simulated using the white noise method. Particles with $3<J_{x}+0.6 \cdot J_{y}<5$ were removed before simulation to produce the situation in Figure 1. The PIC simulations include about $10^{8}$ particles for 6000 turns.

Now if we negate this expression we get:

$$
\operatorname{Im}\left(R_{i}(\Omega)\right) \neq 0 \Rightarrow \exists J_{x}, J_{y}: \frac{\mathrm{d} \psi\left(J_{x}, J_{y}\right)}{\mathrm{d} J_{i}} \neq 0 \wedge \omega_{i}\left(J_{x}, J_{y}\right)=\Omega
$$

This reads as: where $\operatorname{Im}\left(R_{i}(\Omega)\right) \neq 0$, there must be a $\frac{J_{i} \mathrm{~d} \psi}{\mathrm{d} J_{i}} \neq 0$. We can neglect the points in which $\psi=0$ but $\frac{J_{\mathrm{i}} \mathrm{d}}{\mathrm{d} J_{i}} \neq 0$ and therefore say: Where $\operatorname{Im}\left(R_{i}(\Omega)\right) \neq 0$ there is a particle with $\omega_{i}=\Omega$.

We cannot conclude that $\operatorname{Im}\left(R_{i}(\Omega)\right)=0$ means $J_{i} \psi=0$ because $\frac{J_{i} \mathrm{~d} \psi}{\mathrm{d} J_{i}}=0$ can be true for $\psi>0$. However we can say that, in the case that the tune shift is a monotonous function of $J_{x}$ and $J_{y}$ even if there is an area, a hole, for which $\frac{J_{i} \mathrm{~d} \psi}{\mathrm{d} J_{i}}=0$ and therefore the BTF is 0 , somewhere on the other side of the hole $\frac{J_{i} \mathrm{~d} \psi}{\mathrm{d} J_{i}} \neq 0$. Otherwise the particle number would become infinite. This can also be observed in the example BTF in Figure 1. If the gradient is very small or the tune as a function of amplitude is not monotonous, the method may not recover the full tune spread, either because the signal is lost in noise or because the tune as a function of amplitude has a maximum in an area where $\frac{\mathrm{d} \psi}{\mathrm{d} J_{i}}=0$.

In real-life scenarios $\operatorname{Im}\left(R_{i}\right)$ will rarely be exactly zero, be it due to numerical accuracy when numerically solving Equation 4, noise due to finite particle num- 
ber in BTF simulation or noise in measurement. Additionally Landau-damping from other sources than tune spread in-plane can play a role, for example damping coupled in from the other plane or the other bunch in beam-beam interactions. Therefore we propose to weaken the condition to assume particles where

$$
\left|\operatorname{Im}\left(R_{i}\right)\right|>t
$$

with $t<1$. This allows to adjust $t$ to compensate for the unwanted contributions and reduce the detected area to where Landau damping from tune spread is dominant. In our simulations and measurements this still gives a good estimate for the frequencies at which there are incoherent particle tunes.

The method can be modified to demand

$$
\left|\operatorname{Im}\left(R_{i}\right)\right|>t\left|R_{i}\right|
$$

and in this form (therefore using $\left|\operatorname{Im}\left(R_{i}\right) R_{i}^{-1}\right|$ instead of $\left|\operatorname{Im}\left(R_{i}\right)\right|$ in the following) is especially strong when investigating the incoherent tune spread in the presence of coherent modes such as the $\pi$ and $\sigma$ modes observed in coherent beam-beam interaction in one interaction point. It is also more suitable for evaluation of particle tracking simulation data where we found the amplitude of the disturbance from the macro particles granularity scales with the signal amplitude.

We are generally interested in the tune spreads of uniform Gaussian beams and therefore our quantity of interest is the width of the interval on which Equation 18 or Equation 19 hold. Figure 3 demonstrates this for a BTF calculated numerically evaluating Equation 4 for a Gaussian beam under tune shift from a defocusing Gaussian charge distribution. In the figure the interval recovered by the threshold method is compared visually with the actual tune distribution. In the rest of this paper we will refer to the method introduced in this section as threshold method. 

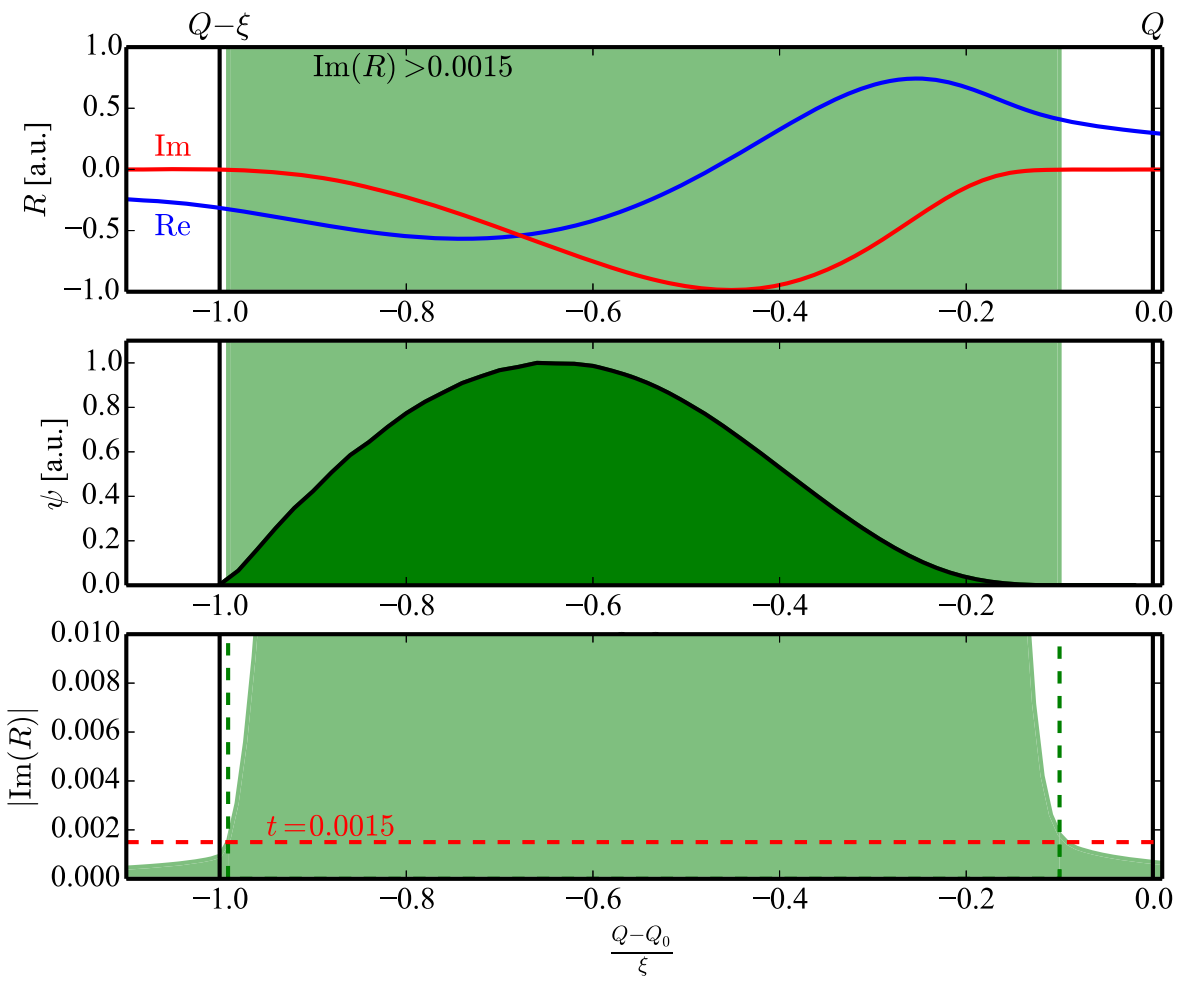

Figure 3: Illustration of how to use the threshold method to determine the tune spread for an analytic BTF, Equation 4 was solved numerically for a beam interacting with a defocusing a Gaussian charge distribution (defocusing electron lens). The area where $|\operatorname{Im}(R)|<t$ is shaded green in the plots of $R$ and $\psi$, and corresponds to the frequencies detected by the threshold method for a threshold of 0.0015 . The beam-beam parameter $\xi$ (left limit of tune width) and the machine tune $Q$ are indicated in the plot. In a magnified plot of $|\operatorname{Im}(R)|, t$ is indicated, and the edges of the recovered tune spread are indicated by green dashed lines. $t$ was chosen to be just above the baseline caused by the method used to numerically solve the BTF integral. The edges of the distribution are most sensitive to the choice of $t$. For the Gaussian beam, the edges correspond to regions where either $J_{x}$ or $\mathrm{d} \psi / \mathrm{d} J_{x}$ is small, so this is not surprising. Still, $t$ between 0 and 0.1 produce results with errors below $20 \%$ of the tune spread. Note also that $\operatorname{Im}(R)$ unlike in the case of chromaticity from Equation 3 is not proportional to $\psi$ in the direction of the BTF on the horizontal tune, anymore. 


\subsubsection{Choice of thresholds}

In this section and wherever we give explicit values for a threshold we assume the BTF is normalized to the value at the betatron peak, this makes comparison of thresholds more convenient. To determine the threshold necessary for the noise level in a given scenario, one chooses an interval outside the tune distribution and outside the area of other additional damping sources (for example the beam-beam interaction coupling Landau Damping from the other the other beam). To identify the total contribution of noise independent of its origin one takes the BTF for such a non-perturbed interval and calculates

$$
a_{\text {noise }}=\max \left(\left|\operatorname{Im}\left(R_{i}\right)\right|\right)
$$
ing can be identified in measurement as discussed in Section 1.7. The method used for evaluation of the measurement is identical to the one in simulation and shown in Figure 4 and is therefore not discussed separately. 
Knowing the shape of $R_{i}$ from analytic considerations for an expected tune distribution allows to correct for the choice of a threshold in simulation or measurement by determining a correction factor that applies for a chosen threshold $t$. If the threshold is chosen to be for example 0.1, one calculates the recovered width $\Delta Q_{\text {threshold }}$ with a threshold of 0.1 for the analytic BTF. For the analytic example one knows the actual tune shift $\Delta Q_{\text {real }}$. To correct for the reduction of tune spread in measurement due to a finite threshold, one multiplies the measured width of the betatron peak $\Delta Q_{\text {meas }}$ at the threshold $t$ with $\Delta Q_{\text {real }} / \Delta Q_{\text {threshold }}$.

\subsection{Fit method for determination of tune spread}

If the shape of the nonlinearity is known and only the width of the tune spread is needed, for example when one knows from another source that the beam size of an electron lens is matched and the beams are Gaussian, one can fit measured BTFs against Equation 4 with the $c$ and $\xi_{\mathrm{bb}}$ (part of $\omega_{i}$ ) as free parameters to arrive at the strength of the interaction. This method is applicable when one has good knowledge of both the $\omega_{i}$ and $\psi$ to be expected.

\subsection{Usage and limitations of the threshold method}

$\operatorname{Im}\left(R_{i}(\Omega)\right)$ becomes nonzero when the oscillation is damped at $\Omega$. Detecting the tune spread works when Landau damping due to tune spread is the dominant source of damping. The threshold method will highlight all frequencies where damping takes place regardless of the source of the damping. Raising the threshold allows to only see the regions with strongest damping. Still this damping might be from another origin than tune spread. Therefore one has to think about how to identify damping from other sources. One possible source of damping is coupling of Landau damping from the other plane or other beam. If one wants to distinguish damping due to tune spread from coupled damping from the other plane, changing the tunes and observing subsequent changes in the BTF allows for identification of coupled-in damping. Another source of damping could be an active damping system, which should be switched off for 


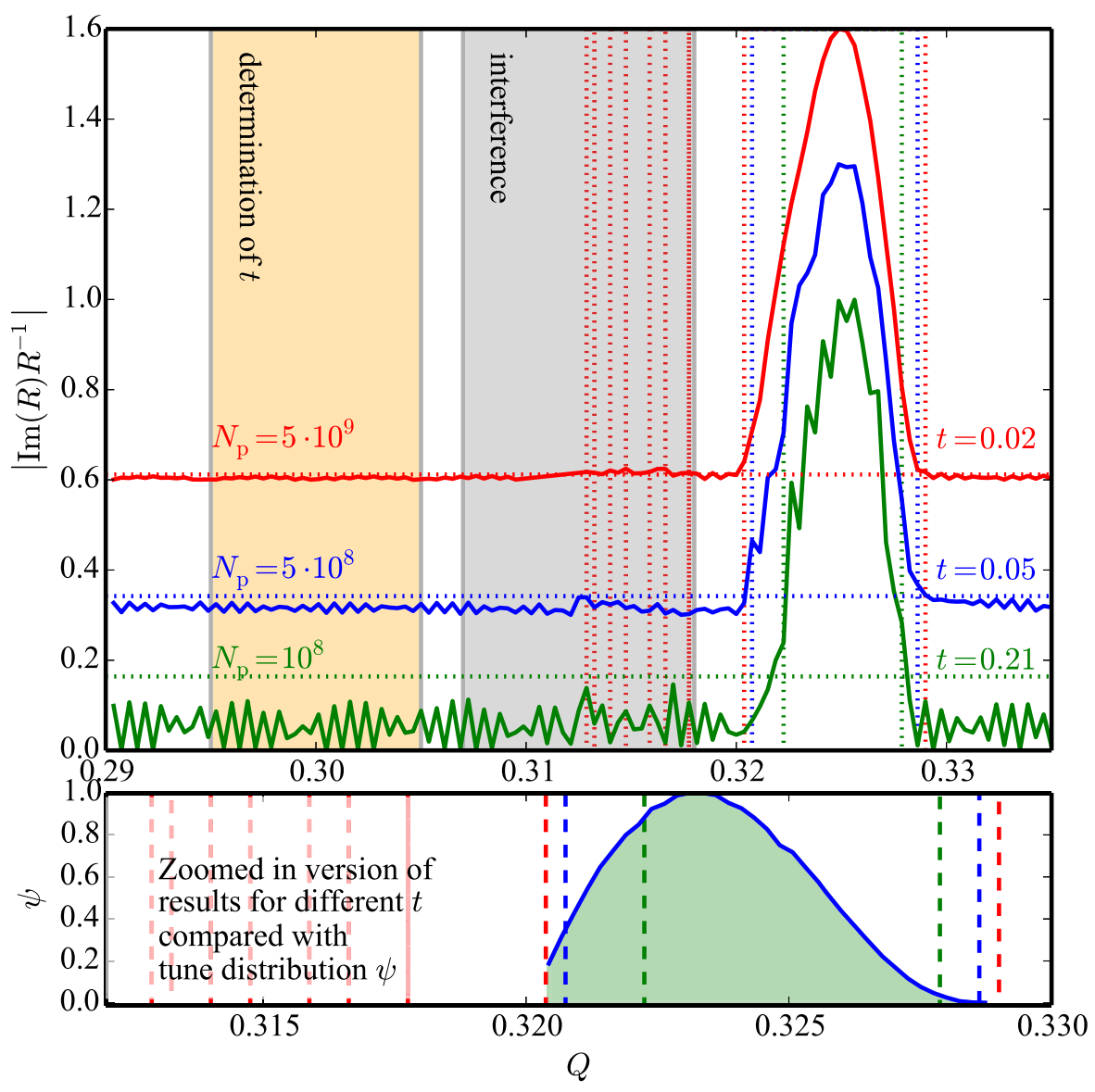

Figure 4: Example application of the threshold method on simulated BTF. The plot shows simulated BTF of a beam disturbed by a defocusing Gaussian charge distribution (defocusing electron lens) for three different macroparticle numbers $N_{\mathrm{p}}$ with otherwise identical beam paramters. In each of the BTFs the threshold was determined by taking $a_{\text {noise }}$ from Equation 20 in the damping-free area shaded in yellow and setting $t=1.5 a_{\text {noise }}$. The thresholds are shown by the dashed horizontal lines, the area recovered by the threshold method lies between the dashed vertical lines. The damping from interplane coupling is shaded in grey. The actual tune distribution from the simulation is shown in the bottom. Note that the plot of $\psi$ has a horizontally magnified scale. One can see that the lower disturbance for the higher simulated particle numbers allows for choosing a lower threshold for better reconstruction of the tune spread. For $N=5 \cdot 10^{9}$ (red dashed lines) the tune spread is recovered with the best accuracy. 
the BTF measurement if the machine is sufficiently stable. Another exception is the presence of coherent modes and will be discussed in later sections.

The question might arise how well the method can detect tune spread in a halo. Unfortunately the particle density and its derivative in the halo will be low apart from possibly the edge of the halo. We are pessimistic about the threshold methods sensitivity for tune spread of halo particles as the low particle density derivative in a smooth halo could only be detected using very low thresholds $t$ and its contribution is likely to be dominated by noise and other sources of damping. However if the halo has structure leading to high $\mathrm{d} \psi / \mathrm{d} J_{i}$ it can become possible to identify tunes at which the density derivative is high. To identify contribution of the halo to the BTF one can compare the BTF before 320 and after scraping of the halo. Changes in $\operatorname{Im}\left(R_{i}(\Omega)\right)$ due to scraping likely reflect particle tunes at $\Omega$.

To estimate the feasibility of studying the halo using BTFs, the contribution of the halo to the BTF can be computed by solving Equation 4 for the assumed particle distribution. By comparing the threshold needed for detection to the typical noise level observed in BTF measurements in the machine one can quickly estimate if the effect would be detectable in the BTF. A method to find the suitable threshold for a measurement system based on the noise level in BTF measurements was described in Section 1.5.1.

The worst-case scenario for the method are beams with (near-)constant particle density in significant fractions of $J_{x}, J_{y}$ space. In the extreme case of constant particle density for an area in $J_{x}, J_{y}$ and 0 outside, the edge of the particle distribution will be the only part detected by the threshold method.

Nevertheless the method will give a good estimate of tune spread whenever the beam is Gaussian or at least of non-constant phase space density, the particle tune is a monotonous or at least smooth function of amplitude and the amount of damping from coupling or other sources is low. All of these conditions can be found in well-adjusted machines. The method will work for coasting beams and, under the conditions discussed in the rest of this paper for bunched beams. 


\section{Simulation model}

340 how BTF are measured, then we describe how we simulate them. We cover different ways of modeling the BTF in simulation. To be clear what effects we take into account in simulation we briefly mention the way we implemented the beam-beam effect and the electron lens. Apart from that we use a sinusoidal 345 RF together with chromaticity to account for synchrotron motion. We validate simulation model and analytic expectation against each other. We then go on to discuss the applicability of the analytic model for coasting beams to bunched beams of low synchrotron frequency, where synchrotron motion has a low influence on the BTF.

\subsection{BTF Measurement setup}

For BTF measurements in the machine, a transverse kicker is used. The kick signal can be white noise (white noise BTF) or a sinusoidal excitation which is then swept over the frequencies of interest (coherent BTF) [1]. The transverse response of the center of charge of the beam is recorded using a pick-up. For 355 both, the BTF is then calculated by computing Equation 1 using the kicker and response signals. In RHIC, BTFs are taken as coherent BTFs at baseband.

\subsection{BTF models in PIC codes}

The two types of BTF can be simulated using PIC codes. For both at chosen locations of the lattice the particles receive a kick in the transverse direction in the plane where the BTF is taken. We operate at baseband, therefore our excitation frequency is below the revolution frequency. We are in a machine where the length of a bunch is much smaller than the circumference. Thus for the simulation we assume that all particles in one bunch receive the same kick amplitude. The kick and response over time is analyzed to compute the BTF. 365 We implement both the coherent and white noise BTF.

For coherent BTFs $[14,15]$ the simulation is done one frequency sample at a time. The drive signal is sinusoidal and the simulation is done for different drive 
frequencies. For each frequency sample the response amplitude is calculated using a discrete Fourier transform at the frequency of the drive signal. The code computes the BTF for the frequency sample according to Equation 1. For parallelization several instances of the code can be used to compute the BTF at different excitation frequencies at the same time. Attention should be paid to avoid nonlinear responses. We tried to keep the maximum beam oscillation amplitude below $1 \%$ of the RMS beam width in simulation. To avoid transient signals from the previous excitation frequency, at each excitation frequency the simulation resets the particle distribution to the initial value before excitation.

For white noise BTF the kicker signal is either white noise or band-limited white noise. This method has the advantage that the result can be used already during the computation and the frequency resolution improves over time as more turns contribute to the BTF. The disadvantage is that in the computation of the BTF, the excitation noise amplitude $D(\omega)$ contributes in the denominator in the computation of the BTF (Equation 1).

If the excitation noise amplitude $D(\Omega)$ is very small for a frequency (as will happen for white noise), even small motions of beam center of charge $A(\Omega)$ that are not, in fact a response to the driving force will be divided by nearly zero and give a large but false contribution to the calculated BTF. A weighted averaging over a range of different realizations of the noise can be used to account for this effect. The assumption made in the definition of the BTF is that the response scales linearly with the driving term. Thus different driving terms $D_{i}$ linearly combined will lead to their responses $A_{i}$ linearly combined. We employ an averaging process to combine the BTF from a number of different driving noise realizations $D_{i}$ and responses $A_{i}$ by weighting the $A_{i}$ and $D_{i}$ with $D_{i}^{*}$. This is permissible since from $R=\frac{A}{D}$ follows that $R=\frac{A^{\prime}}{D^{\prime}}$ with $A^{\prime}=A D^{*}$ and $D^{\prime}=D D^{*}$. We now sum over the realizations of the weighted responses $R_{i}^{\prime}$ and weighted driving terms $D_{i}^{\prime}$ arriving at:

$$
R(\Omega)=\frac{\sum_{i} A_{i} D_{i}^{*}}{\sum_{i} D_{i} D_{i}^{*}}
$$

In this version the low driving amplitudes only contribute little to the sum in 
the denominator. Therefore we get a significantly better signal to noise ratio than we would have for a non-weighted averaging of the BTF realizations.

To test the simulation model against the analytic expectations, the BTF of beams with an electron lens as the sole source of tune spread were simulated. The electron lens in simulation was approximated by a kick corresponding to the fields of a Gaussian transverse charge distribution and compared to the analytic result from Equation 4. They are in agreement, an example was already shown in Figure 2.

Furthermore the BTF simulation model was validated on a coasting beam with chromaticity as the source of tune spread and the results were in agreement with Equation 3. We conclude from this that the simulation model used and the threshold method are valid independent from the source of incoherent tune spread.

\subsection{The model of the beam-beam effect used in our simulations}

For PIC simulations we use an idealized model: on top of the PATRIC [16] code we implemented a model of the beam-beam effect [13]: We run one instance of the code for each bunch (in the following we will, for the sake of brevity use the word bunch for the process simulating one bunch). Whenever bunches arrive at an interaction point (IP), they compute their transverse electric field using a two-dimensional FFT-based poisson solver under the assumption of open boundary conditions. They exchange the transverse electromagnetic fields via the message passing interface (MPI) with their collision partner. The bunches modify the momentum coordinate of their particles according to the value of the received field at the location of the particle. The beam-beam interaction is reduced to a single kick. For our measurement setup at RHIC there are no long-range interactions. After the beam-beam interaction, the translation to the next IP is done using a linear transfer map calculated using MADX on the RHIC lattice [17]. We justify the reduction of the lattice to a linear transfer map by the beam-beam interaction being the dominant nonlinearity in the transverse direction at the machine conditions of concern. 
2.4. Synchrotron oscillations for relativistic bunches with small synchrotron frequency

The analytic results are on their own only valid for coasting beams in absence of coherent modes. We wanted to verify that the synchrotron motion has a negligible influence on the BTF shape for synchrotron periods of the order of magnitude of the BTF measurement time and compensated but nonzero chromaticity, common conditions in high energy machines. To verify that this is the case, we ran simulations of coasting beams and bunched beams and compared them with each other. The BTF did not show notable difference for typical high energy collider parameters where the betatron tune oscillation due to synchrotron motion is a minor contribution to the tune spread. A table of the simulation parameters can be found in Table 1

Table 1: Simulation parameters for the evaluation of the assumption of coasting beams in beams with low synchrotron frequency and low chromaticity. For these parameters no notable dependence of the shape of the BTF in dependence of synchrotron frequency was observed. Typical RHIC high energy proton parameters are shown for comparison.

\begin{tabular}{lrr}
\hline & RHIC & Simulation \\
\hline Chromaticity & $\approx 0-4$ & $0-4$ \\
Momentum spread & $\approx 2 \cdot 10^{-4}$ & $2-8 \cdot 10^{-4}$ \\
BTF sample time & $\approx 30 \mathrm{~ms}$ & $30-50 \mathrm{~ms}$ \\
Synchrotron period & $\approx 30 \mathrm{~ms}$ & $15 \mathrm{~ms}, 30 \mathrm{~ms}$, coasting \\
\hline
\end{tabular}

\subsection{Coherent modes}

We used the simulation model to check for the applicability of the threshold method to beams undergoing strong-strong beam-beam interactions with coherent beam-beam modes. With beam-beam there are coherent modes and depending on the machine properties some of them will fall into the incoherent spectrum[18]. It is to be expected that at coherent modes the imaginary part 
becomes non-zero for a small intervall of tunes. Nevertheless we expect that still the imaginary part stays nonzero in the region of the incoherent tune spread. So the threshold method may still give a usable estimate for the tune spread if one is able to identify coherent modes in the BTF as such.

Simulation results can be found in Figure 5. In the simulation with two 450 bunches colliding in one interaction point, the coherent modes are easily identified and the tune spread can be recovered. For identification of the coherent modes it is necessary to have the BTF in a frequency resolution sufficient to resolve the coherent modes and separate them from the incoherent spectrum. Additionally one should know their number and approximate positions. For the two-bunch case presented here, the $\sigma$-mode is expected at the lattice tune and the $\pi$ mode is separated from it by the beam-beam parameter $\xi$ multiplied with the Yokoya factor [18]. 

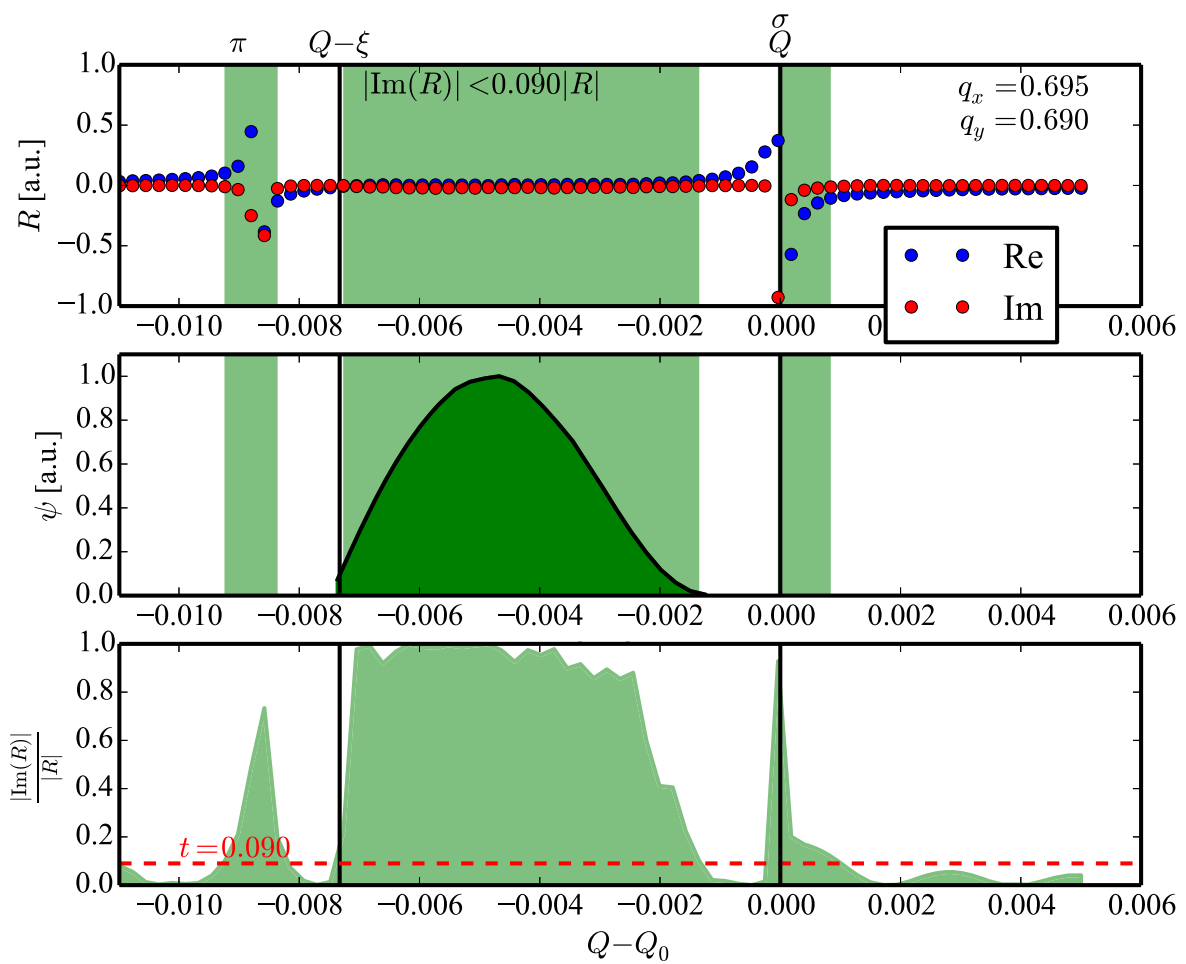

Figure 5: Threshold method for simulated horizontal BTF of two colliding bunches with coherent $\pi$ and $\sigma$ modes due to the beam-beam effect. See also the caption of Figure 3 for more details on what the plots show. Even though the BTF is dominated by the coherent modes from the Beam-Beam effect in the BTF, the threshold method nicely recovers the tune spread plus some additional area at the coherent modes (where the imaginary part becomes finite because of coherent effects). The coherent modes can easily be identified in this scenario and the incoherent tune spread can be read from the threshold method. The area fulfilling the inequation is shaded in the Plots of the BTF $R$ and the tune density $\psi$ and after neglecting $\pi$ and $\sigma$ modes nicely covers the spread of incoherent tunes. Here we used Equation 19 since we are dealing with simulation data and coherent modes. 


\section{Measurements}

In this section we discuss measurements of BTFs with the beam-beam effect.

460 second objective was to test the threshold method described above and compare it with the analytic model.

We first present the measurement conditions and how we suppress coherent modes in measurements with the beam-beam effect. We compare the measured BTF to our analytic results by means of fitting against Equation 4. Then we compare the beam-beam parameter recovered from the fit to the result obtained using the threshold method.

\subsection{Coherent modes}

Equation 4 is valid in absence of coherent modes. In the measurement we wanted to assess our approximation of using the coasting beam formalism for a bunched beam with low synchrotron frequency At the time of measurement there was no electron lens device in operation to test our theory. The incoherent tune spread caused by the beam-beam effect is of the same shape as that of an electron lens. Therefore one might think of the beam-beam effect as a suitable source interactions often show coherent beam-beam modes [18]. These can deform the spectrum and will not be present in pure electron lens operation. To compare Equation 4 with a beam-beam measurement we had to suppress coherent modes: Alexahin [19] gives two ways of making the coherent modes disappear: Splitting the tunes of the two rings and splitting the intensities of the beams. In both cases coherent modes move into the incoherent spectrum and become Landaudamped, therefore the process can lead to residual deformation of the BTF.

\subsection{Measurement conditions}

For comparison with the analytic expectation we needed to ensure the Landaudamped coherent modes would not significantly distort the spectrum for our measurement conditions. We decided to measure the BTF of the weak beam 

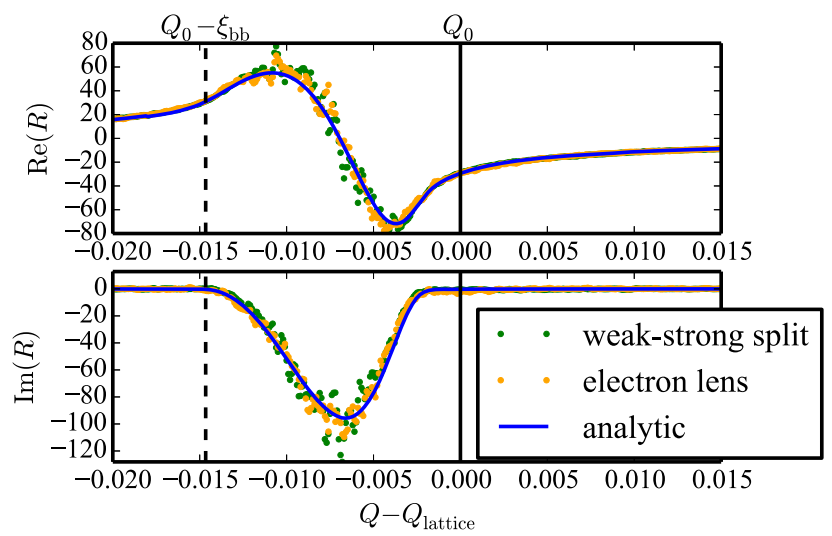

Figure 6: Simulated BTFs, comparison between the BTF of the weak beam in a weak-strong beam-beam interaction with split tunes and a (defocusing) electron lens. The two results are approximately the same. The fit of Equation 4 against the BTF correctly recovers the tune spread, its result and the analytic expectation correspond and are shown in the plot.

in a weak-strong beam-beam interaction similar to an electron lens. We chose beams in which one beam consisted of bunches of just $\frac{1}{10}$ the intensity of the other. In ref. [19] Alexahin showed that an intensity ratio between colliding bunches below $1 / 2$ should be sufficient to move the $\pi$-mode into the incoherent spectrum and Landau-damp it. In this case the beam-beam $\sigma$ mode still persists. The $\sigma$ mode can then be removed by splitting the tunes of the two machines $\left|Q_{\text {blue }}-Q_{\text {yellow }}\right| \approx .03$. In simulation this setup shows agreement with the scenario of an electron lens as shown in Figure 6.

\subsection{Measurement}

To test our analytic theory and the threshold method on a measurement, we took BTFs in a dedicated beam time during the RHIC 2013 proton run. The blue ring was filled with bunches of $1.8 \cdot 10^{11}$ particles per bunch. The yellow ring was filled with bunches of different intensity: Six equally spaced bunches of normal intensity to provide a sufficient signal for the tune feedback on the ramp and 22 Bunches of $10 \%$ intensity as weak bunches for the BTF measurement. A cartoon of the filling scheme can be found in Figure 7. At the beginning of the 
experiment, the beams were colliding in two interaction points at the PHENIX and STAR experiments. First a BTF of the entire beam was taken. Then a gated BTF of the 22 weak bunches was taken. The beams were separated in one of the two interaction points (PHENIX). Again, a gated BTF of the weak bunches was taken. To split the tunes, the fractional tune of the strong beam was increased. A final set of gated BTF of the weak bunches was taken, then the beam was dumped.

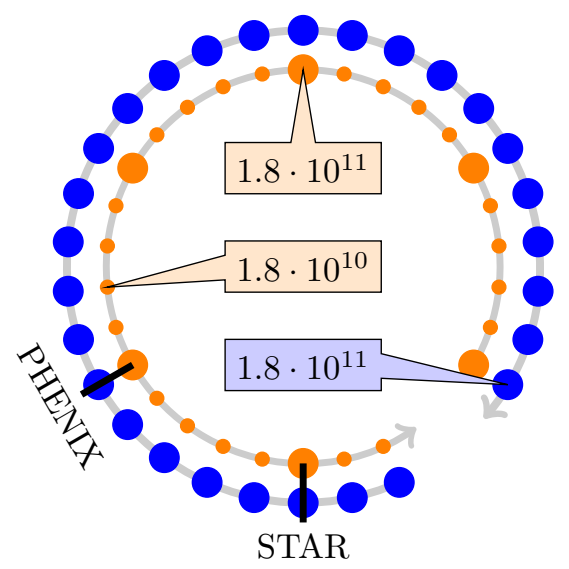

Figure 7: Bunching scheme for the experiment. Initially the beams were colliding in STAR and PHENIX. Later the beams were moved out of collision in PHENIX. The measurement for electron-lens like conditions was done using gated BTFs on the low intensity bunches in the yellow ring. The 6 high intensity bunches are necessary to have signal intensity for the tune feedback on the ramp. They were also used to determine the beam-beam parameter via the $\pi$ and $\sigma$ mode positions in the strong-strong collisions.

\subsection{Results and discussion}

The beam-beam parameter $\xi$ in the horizontal plane was determined from the distance of the $\pi$ and $\sigma$ modes observed in the BTF of the strong bunches in the yellow beam in the presence of two beam-beam interactions. The beambeam parameter was determined to be 0.017. The vertical BTF did not show coherent modes and the beam-beam parameter could not be determined from 
Table 2: Beam-Beam parameter calculated for the two methods of evaluating the measured BTF: fitting Equation 4 against the BTF and the threshold method introduced in this paper. The beam-beam parameter in the horizontal plane determined from the distance of the $\pi$ and $\sigma$ modes for strong bunches in the horizontal direction was 0.017. In the vertical plane no coherent beam-beam modes were observed so no additional means of determining the beambeam parameter was available. To make the results comparable, the beam-beam parameter $\xi$ was computed from the results of the threshold-method according to the method given in the text. The expectation is that the beam-beam parameter determined for the case of two interaction is twice as high as for one interaction. Split tune measurements were also done with only one interaction.

\begin{tabular}{lrrr}
\multicolumn{2}{c}{ expected factor } & $\approx \times 0.5$ & \multicolumn{1}{c}{$\times 1$} \\
\hline & two interactions & one interaction & split tunes \\
\hline$\xi_{h, \text { threshold }}$ & 0.0179 & 0.095 & 0.093 \\
$\xi_{h, \text { fit }}$ & 0.0178 & 0.0093 & 0.0086 \\
$\xi_{v, \text { threshold }}$ & 0.0273 & 0.0170 & 0.0157 \\
$\xi_{v, \text { fit }}$ & 0.0275 & 0.0171 & 0.0156 \\
\hline
\end{tabular}

their position for this reason. The blue beam was approximately round as measured via ionization profile monitor, therefore we would expect that the beam-beam parameter the yellow beam sees should be similar in both planes.

The analytic equation for the BTF (Equation 4) with tune spread due to a Gaussian lens was fitted to the measured BTF of the weak bunches in the yellow beam in both planes. The fit showed a good approximation to the measured BTF giving confidence in the assumption that due to low synchrotron frequency we can treat the BTF in the limit of coasting beams. The beam-beam parameter determined from the fit for one and two beam-beam interactions can be found in Table 2. We see good agreement between our two methods. Furthermore, our expectation that upon removing one of two interactions the tune spread and with it the beam-beam parameter is reduced by a factor of 2 is confirmed by the measurement in both planes. 

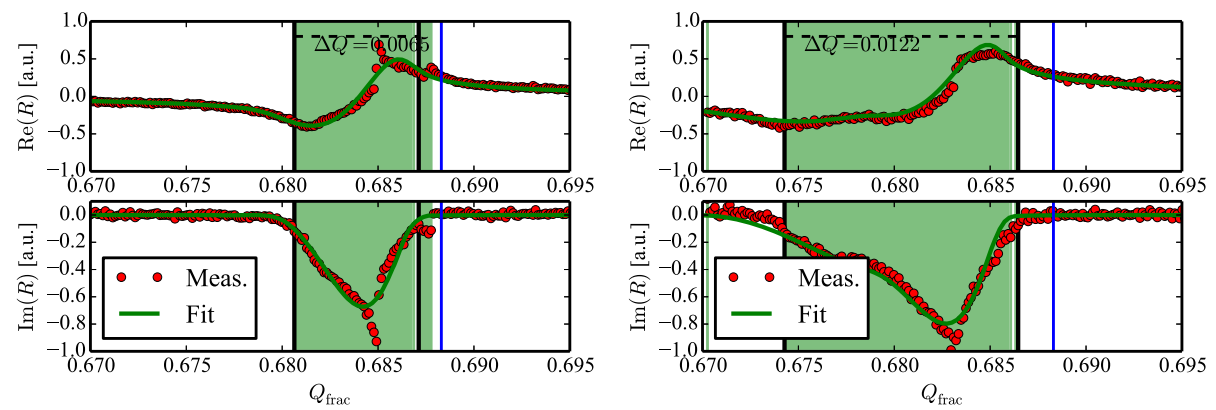

(a) BTF and threshold tune width for beams col-

(b) BTF and threshold tune width for beams colliding in one IP

liding in two IPs

Figure 8: Results for the width of the tune spread from the threshold method are given in the respective plots. The greeen area is where the threshold condition is fulfilled, the chosen threshold is 0.1. The blue line denotes horizontal tune of the machine at the time of the measurement, the green line is a fit of Equation 4 to the data.

The vertical plane shows a multi-peak structure the source of which is not entirely understood. However we can still observe the expected behaviour that the width of the main peak roughly halves when moving the beams out of collision in one IP. Therefore we applied the fitting even though several unexpected peaks were seen. Figure 9 shows the vertical BTF with collisions in both and only one IP. The BTF and fitted results can be found in Figure 8 and Figure 9 respectively. While the tune spread measured using the fit and the threshold method is in agreement, the question for the source of the large tune spread / beam-beam parameter in the vertical direction remains.

After the measurement it became apparent that two of the weak bunches had a number of collisions different from the others as they were lacking a partner in one IP. Therefore the fits presented here were made against a superposition of responses with the respective numbers of collisions for the evaluation.

\section{Conclusion}

We presented a model for the transverse BTF of relativistic bunched beams with a betatron tune shift from a localized transverse nonlinearity. The aim 

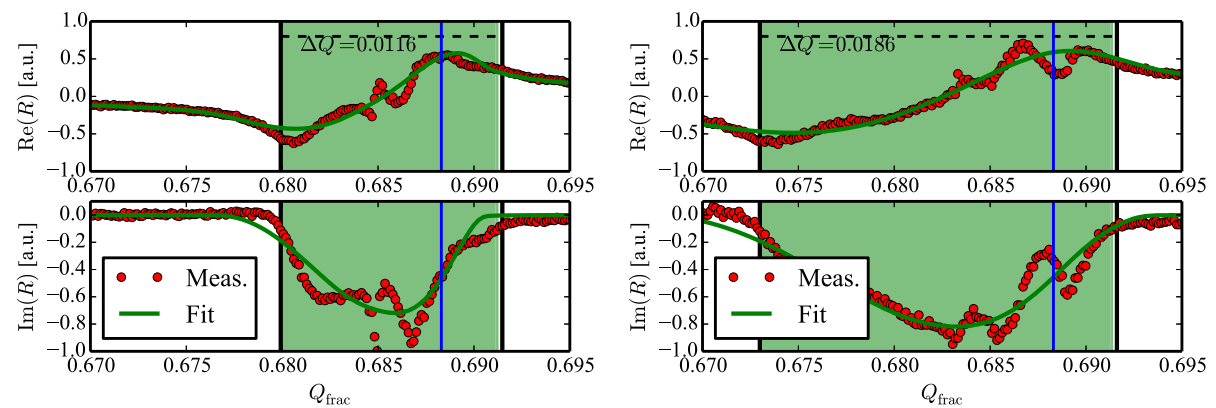

(a) Vertical BTF and threshold tune width for

(b) Vertical BTF and threshold tune width for beams colliding in one IP

beams colliding in two IPs

Figure 9: Results for the beam-beam parameter from the fit of the analytic expressions against the measurement data are given in Table 2. The green line is a fit of Equation 4 to the data. The greeen area is where the threshold condition is fulfilled, the chosen threshold is 0.1. The blue line denotes horizontal tune of the machine at the time of the measurement.

of this work is to retrieve the betatron tune distribution from measured BTFs.

Our simplified analytic BTF model agrees with simulation and measurement results obtained in RHIC. Using our simplified model we point out that the recovery of the tune distribution from the BTF without prior knowledge of the beam distribution and the nonlinearity is impossible. Instead we presented an approach we termed threshold method: Unlike the tune distribution, the tune spread can be recovered from the BTF: it is the area where the imaginary part of the BTF is nonzero. We chose a threshold for the imaginary part of the BTF to account for Landau damping from the incoherent particle tune distribution. This threshold method gives a good estimate for the spread of incoherent particle tunes, but no indication on the shape of the tune distribution. The method is applicable for coasting beams and bunched beams with a nearfrozen synchrotron motion.

We performed simulations including coherent beam-beam interactions which indicate that even in presence of coherent modes the method gives promising results.

Finally we compared the analytic model and the threshold method to BTF 
data obtained in RHIC. The two methods were in agreement with each other and the tune spread expected from the beam-beam parameter measured via coherent beam-beam modes. While the analytic model for the BTF relies on case of a known amplitude dependent tune spread, the threshold method is more general and can be applied even in the presence of coherent modes. For specific machine conditions we would advise to verify its applicability for the specific situation in simulation before using it in measurement. Furthermore we consider the method to be applicable to coasting beams in general and to bunched beams under the condition of frozen or near-frozen synchrotron motion.

\section{Acknowledgements}

P. Görgen wishes to thank S. White and M. Blaskiewicz for their support, especially during the early phase of the work.

\section{References}

[1] J. Borer, G. Guignard, A. Hofmann, E. Peschardt, F. Sacherer, B. Zotter, Information from beam response to longitudinal and transverse excitation, Nuclear Science, IEEE Transactions on 26 (3) (1979) 3405-3408.

URL http://cdsweb.cern.ch/record/133210http://ieeexplore.

[2] M. Gasior, R. Jones, The principle and first results of betatron tune measurement by direct diode detection, LHC-Project-Report 853, Tech. Rep. August 2005, CERN, Geneva (2005).

URL http://cdsweb.cern.ch/record/883298

[3] S. Paret, V. Kornilov, O. Boine-Frankenheim, Transverse Schottky and beam transfer function measurements in space charge affected coasting ion beams, Physical Review Special Topics - Accelerators and Beams 13 (2) 
(2010) 1-7. doi:10.1103/PhysRevSTAB.13.022802.

URL http://link.aps.org/doi/10.1103/PhysRevSTAB.13.022802

[4] R. Singh, O. Boine-Frankenheim, O. Chorniy, P. Forck, R. Haseitl, W. Kaufmann, P. Kowina, K. Lang, T. Weiland, Interpretation of transverse tune spectra in a heavy-ion synchrotron at high intensities, Physical Review Special Topics - Accelerators and Beams 16 (3) (2013) 034201. doi:10.1103/PhysRevSTAB.16.034201.

95 URL http://link.aps.org/doi/10.1103/PhysRevSTAB.16.034201

[5] W. Fischer, X. Gu, S. M. White, Z. Altinbas, D. Bruno, M. Costanzo, J. Hock, A. Jain, Y. Luo, C. Mi, R. Michnoff, T. A. Miller, A. I. Pikin, T. Samms, Y. Tan, R. Than, P. Thieberger, First Experience with Electron Lenses for Beam-beam Compensation in RHIC, TUYA01, in: Proceedings of $5^{\text {th }}$ International Particle Accelerator Conference, Dresden, 2014.

URL http://www.jacow.org

[6] V. Shiltsev, Y. Alexahin, K. Bishofberger, V. Kamerdzhiev, G. Kuznetsov, X.-L. Zhang, Experimental Demonstration of Colliding-Beam-Lifetime Improvement by Electron Lenses, Physical Review Letters 99 (24) (2007) 244801. doi:10.1103/PhysRevLett.99.244801.

URL http://link.aps.org/doi/10.1103/PhysRevLett.99.244801

[7] V. N. Litvinenko, G. Wang, Compensating tune spread induced by space charge in bunched beams, Phys. Rev. ST Accel. Beams 17 (2014) 114401. doi:10.1103/PhysRevSTAB.17.114401.

URL http://link.aps.org/doi/10.1103/PhysRevSTAB.17.114401

[8] G. Stancari, A. Valishev, R. Bruce, S. Redaelli, A. Rossi, B. Savachua, Electron Lenses for the Large Hadron Collider, TUOBA01, in: Proceedings of $5^{\text {th }}$ International Particle Accelerator Conference, Dresden, 2014.

URL http://www. jacow.org

[9] J. Berg, F. Ruggiero, Landau damping with two-dimensional betatron tune spread, CERN-SL (AP) 71 (1996) 1996. 
[10] H. G. Hereward, Landau damping by non-linearity, Tech. Rep. CERNMPS-DL-69-11, CERN, Geneva (Jul 1969).

[11] K. Ng, Physics of Intensity Dependent Beam Instabilities, World Scientific, Singapore, 2006.

[12] A. Burov, V. Lebedev, Transverse instabilities of coasting beams with space charge, Physical Review Special Topics - Accelerators and Beams 12 (3) (2009) 034201. doi:10.1103/PhysRevSTAB.12.034201.

URL http://link.aps.org/doi/10.1103/PhysRevSTAB.12.034201

[13] A. W. Chao, K. H. Mess, M. Tigner, F. Zimmermann (Eds.), Handbook of Accelerator Physics and Engineering, 2nd Edition, World Scientific, Singapore, 2012.

[14] H. Kim, T. Sen, Beam-beam simulation code BBSIM for particle accelerators, Nuclear Instruments and Methods in Physics Research Section A: Accelerators, Spectrometers, Detectors and Associated Equipment 642 (1) (2011) 25-35. doi:10.1016/j.nima.2011.03.059.

URL http://linkinghub.elsevier.com/retrieve/pii/ S0168900211007017

[15] T. Pieloni, W. Herr, A. Bay, L. Rivkin, A study of beam-beam effects in hadron colliders with a large number of bunches, Ph.D. thesis, Ecole Polytechnique, Lausanne (2008).

[16] O. Boine-Frankenheim, V. Kornilov, Implementation and Validation of Space Charge and Impedance Kicks in the Code Patric for Studies of Transverse Coherent Instabilities in the Fair Rings, in: Procedings of ICAP 2006, 2006, pp. 267-270.

[17] S. Tepikian, private communication (2012).

[18] K. Yokoya, K. Haruyo, Tune Shift of Coherent Beam-Beam Oscillations, Particle Accelerators 27 (1990) 181-186. 
[19] Y. Alexahin, A study of the coherent beam-beam effect in the framework of the Vlasov perturbation theory, Nuclear Instruments and Methods in Physics Research Section A: Accelerators, Spectrometers, Detectors and Associated Equipment 480 (2-3) (2002) 253-288. doi:10.1016/S0168-9002(01)01219-0.

URL http://linkinghub.elsevier.com/retrieve/pii/ S0168900201012190 